\title{
sciendo
}

DOI 10.2478/sbe-2020-0017

SBE no. 15(1) 2020

\section{US ECONOMIC POLICY UNCERTAINTY AND GCC STOCK MARKET PERFORMANCE}

\author{
SAEED ABDULLAH \\ The University of Arizona, USA
}

\begin{abstract}
:
The study evaluates the effect of economy policy uncertainty of US on gulf cooperation council (GCC) countries' stock market returns. The GCC countries are Saudi Arabia, Qatar, UAE, Kuwait, Bahrain and Oman. Granger Causality Tests (GCT) was done primarily to evaluate if economy policy uncertainty granger cause on GCC stock market returns. The analysis established that oil prices granger cause stock market returns for Saudi Arabia, Kuwait and UAE; the same is not true on changes in economic policy uncertainty of US cause on the stock market returns. Changes in economy policy uncertainty in US granger causes on stock market returns of Bahrain. On the other hand, economy policy uncertainty in US does not cause stock market returns in Qatar, UAE, Kuwait and Saudi Arabia. Vector Autoregression (VAR) analysis establishes that economy policy uncertainty in US negatively responds to the stock market returns of the GCC countries.
\end{abstract}

Key words: GCC, EPU, Granger Causality Test, Vector Autoregression (VAR)

\section{Introduction}

There has been a booming economic growth among the Gulf Cooperation Council countries (GCC). According to institute of international finance (IIF) forecasts, the overall economic growth rate in GCC countries is forecasted as 2.3\% and $2.7 \%$ in 2018 and 2019 respectively. The positive economic growth is attributed by rise in oil prices and increased government spending. According to Duenwald and Tamirisa (2018), increase in oil production in the GCC countries the overall economic growth is forecasted at $2.9 \%$ in the year 2019. The positive economic growth induces strong trade ties between the US and GCC countries.

Furthermore, IIF attests that there is high expectations of nonhydrocarbon growth in the GCC countries that will accelerate economic growth to $3.2 \%$ by 2020 (Saxena \& Al-Hadrami, 2017). In this case the fiscal positions among the GCC countries are expected to incur improvement due to increase in oil production. For this reason, oil prices play a significant role in explaining variations in the GCC stock markets. The booming economic growth among the GCC countries plays a significant role in enhancing trade ties between the US and GCC economies (Aloui, Hammoudeh, \& Hamida, 2015). Additionally, economic integration among the GCC countries has significantly led to the increase in 
trade ties with US. According to Boughanmi, Al-Shammakhi \& Antimiani, (2016) GCC is the most organized and large sub regional trade integration in the MENA region and also globally. The major aim that led to formation of GCC integration in the region was to initiate free trade in goods primarily to initiate high levels of cross-national labor and mobility in capital.

Several macroeconomics have documented key roles of spillovers from the US economy to the global economy. US economy is the world largest single economy (Bjørnland, \& Zhulanova, 2018). Evidently, US economy accounts approximately a quarter of the global gross domestic product at market exchange rate. Additionally, the US economy accounts for a fifth of the global foreign direct investment and more than $33 \%$ of the market capitalization. According to Gay (2016), confirms that a surge in US growth rate substantially affects global economy. The surge can result from application of monetary policies to the US economy. In this case, shocks from the US economy are equally transmitted to the rest of world economies through three potential ways (Bekaert et al., 2014). To begin with, fast economy growth in the US economy effectively lifts growth of the trading partners through massive import demands and strengthening spillovers embedded in the trading acts. Secondly, the act leads to financial market development that in turn has positive financial implications to the global markets. US bond and equity markets are widely used globally alongside with the US dollar as the globally recognized currency. Some of the eminent equity market is between US and UK. According to Ahmed, Coulibaly, \& Zlate, (2017) provides rich evidence on the contemporaneous spillover effects between the USA and UK equity markets. Thirdly, US is the leading gas and oil consumer globally. When shocks occur in the US economy ultimately are transmitted to the other equity markets.

Studies confirm that there is a rich history of trading cooperation between US and GCC countries. Evidently, the trading relationship dates back to 1700 s years when Americans seemingly enjoyed commercial activities from the Sultanate of Oman (Commins, 2015). The trading activities were sometimes marred with challenges like wars in the gulf region. However, formation of international bodies like UN has enabled for the restoration. Additionally, formation of gulf council cooperation (GCC) equally enhanced trading ties between US and GCC countries. Oil commodity is another aspect that has led to strong trading ties between US and GCC countries. According to Al-Maamary, Kazem, \& Chaichan, (2017), GCC countries are the greatest oil producers while US is the largest oil consumer thus great oil importer from GCC countries. Concomitant to GCC countries selling oil and USA purchasing oil, this translates to annual billions of dollars to the both parties. This greatly seals the presence of strong economic ties between the US and GCC countries.

Researches have demonstrated effect of macroeconomic variables stock returns and on several financial assets in the global market. According to Mahedi (2012), testifies for the both long and short term relationships among the various macroeconomic variables and stock returns using Johansen Cointegration tests using stock returns from German and UK markets. However, this leaves a room to investigate the casual relationships among the macroeconomic variables using simple correlation analysis. An empirical study conducted by Mohamed Arouri \& Christophe Rault \& Frédéric Teulon, (2014) confirm that 
economic policy uncertainty affects stock markets in USA, Europe, China and GCC countries. In the study panel data was equally applied to attest for the effect of economic uncertainty on the stock markets. In their research they discovered that increase in EPU negatively affects the stock returns.

The primary aims of the study are to evaluate the effect of variations in economic policy uncertainty in USA on the stock markets of gulf cooperation council countries (GCC). The GCC countries are Saudi Arabia, Kuwait, UAE, Qatar, Bahrain and Oman. Secondly, the study evaluates how the stock market of the GCC countries responds to the changes in EPU of USA. Thirdly, the study evaluates whether changes in economic policy uncertainty may affect the stock market returns of the six GCC countries using granger Casuality test.

\section{Method and Data}

The data for the variables "oil prices (Brent) and monthly returns" for the six GCC countries are for the period 1/31/2010-8/31/2018 are sourced from Bloomberg. Data for the economy policy uncertainty (EPU) are obtained from the official online site of EPU located at http://www.policyuncertainty.com.

The main analysis methodology is time series OLS regression model. The response variable is set as stock market of the GCC countries. On the other hand, the explanatory variables included in the analysis are changes in economic policy uncertainty of the US and oil prices (Brent). Equations 1 and 2 are the most preferred models for the analysis to respond to the study objectives.

$$
\begin{aligned}
& R_{i t}=\alpha+\beta \Delta E P U_{i t}+\varepsilon_{i t} \ldots \ldots \ldots \ldots \ldots \ldots \ldots(1) \\
& R_{i t}=\alpha+\beta \Delta E P U_{i t}+\delta O P_{i t}+\varepsilon_{i t}
\end{aligned}
$$

Where;

$\mathrm{R}_{\text {it }}$ is the return on a country's market stock index in month $t$

$\triangle E P U_{i t}$ is change in USA economic policy uncertainty index in month $t$

$\mathrm{OP}_{\text {it }}$ is the return on oil price index in month $t$

$\varepsilon_{i t}$ is the error term in the model

The variable oil prices (Brent)" are used as control variable primarily to control the effect of Oil price effects on GCC stock markets.

\section{Results}

The illustration on table 1 displays descriptive statistics of the GCC countries' stock market and the control variable, Oil Price. Table 2 displays correlation analysis for the stock market variables and the control variable. Evidently, there is lack of relationship among the variables. The first aspect in the analysis was to check whether the series are indeed stationary using ADF tests shown in the appendix section. It was evaluated that the series were indeed non stationary and therefore single differencing was therefore necessary to make the series stationary before OLS regression models are evaluated. The regression models attest for the effect of US economic policy uncertainty to the stock 
markets for the GCC countries. The F-statistics in the respective time series OLS models are used to test whether the fitted models are of good fit for the data. In this regard, hypothesis is set as follows;

$$
H_{0}: \beta_{1}=\beta_{2}=\ldots=\beta_{i}=0 \text { for } i=1,2,3 \ldots
$$

Against

$$
H_{1}: \beta_{1} \neq \beta_{2} \neq \ldots \neq \beta_{i} \neq 0 \text { for } i=1,2,3 \ldots
$$

The illustration on table 3 displays OLS regression output for the first equation where changes in USEPU index is regressed on the $6 \mathrm{GCC}$ stock markets. The analysis establishes that Saudi Arabia- $(F(1,103)=0.3595)$, Kuwait- $(F(1,103)=1.416)$, UAE$(F(1,103)=0.1564)$, Qatar- $(F(1,103)=0.8938)$, Bahrain $-(F(1,103)=0.0662)$ are statistically insignificant at 0.05 alpha level. However, this does not bar the researcher from evaluating the effect of the oil prices and changes in US economic policy uncertainty to the respective GCC economies. OLS without control variable shows that results for the $6 \mathrm{GCC}$ countries are statistically insignificant at 0.05 alpha level.

US economic policy uncertainty has negative effect on the stock markets for Saudi Arabia, Kuwait, UAE, Qatar, Oman and Bahrain. The illustration on table 3 displays the effect of USEPU on the various stock market returns. The analysis establishes that the coefficients for the models are Saudi Arabia- $(\beta 1=-0.9708)$, Kuwait- $(\beta 1=-1.2298)$, UAE$(\beta 1=-0.2626)$, Qatar- $(\beta 1=-1.8831)$, Bahrain- $(\beta 1=-0.0347)$ and Oman- $(\beta 1=-1.5460)$.

To control the effect of Oil Price, the stock markets are regressed on the changes in US economic policy uncertainty, and oil price returns using equation 2 . The illustration on table 4 reveals results for Saudi Arabia. The variables "USEPU, and Brent" has coefficients of $-3195(p$-value $=0.838))$ and $28.5026(p$-value $<0.010)$ respectively. Oil prices have significant effects at 0.05 alpha level. Oil prices (Brent) has positive effect on the stock market. Table 10 reveals results for Kuwait. The analysis establishes that variables "USEPU and Brent" has coefficients of -1.1182 and 4.8861 respectively. The variables are statistically insignificant at 0.05 alpha level. Only oil prices reveal positive effect on Kuwait stock market. Based on table 4, the analysis establishes that the variables "USEPU and Brent" has coefficients of -0.1639 and 4.3215 respectively on UAE stock market. Table 4 illustrates analysis on Qatar stock market. The variables "USEPU and Brent" has coefficients of -1.4398 and 19.4040 respectively. The effect of oil prices is statistically significant at 0.05 alpha level ( $p$-value=0.062). Additionally, the variables "USEPU and Brent" has coefficients of -0.0106 and 1.0550 respectively on Bahrain stock market. USEPU and oil prices reveals statistical insignificance at 0.05 alpha level ( $p$-value $>0.05$ ). Lastly, it is established that the variable USEPU has statistical significant effect of -1.3336 on Oman stock market ( $p$-value $=0.0848$ ) at $10 \%$ significance level. On the other perspective, the variable "oil prices (Brent)" has a positive coefficient of 9.2990 that is statistically significant at 0.05 alpha level ( $p$-value $<0.010$ ). Basically, OLS results with oil prices as control variable for the stock markets Oman, Qatar and Saudi Arabia are statistically significant at 0.05 alpha level. On the other hand, OLS results for UAE, Kuwait and Bahrain stock markets are statistically insignificant at 0.05 alpha level.

Vector autoregression analysis is performed to attest how the control and explanatory variables responds to the stocks of 6 GCC countries. According to Juselius (2006), VAR model is a stochastic model that assist in capturing linear interdependencies 
in various time series. In the analysis, VAR modelling is applied to capture interdependencies among the respective stock markets, the control variable and changes in US economy policy uncertainty. The control variable is oil prices (Brent). Results for VAR analysis results for the 6 GCC countries are displayed in the appendix. The illustrations on tables 23, 24, 25, 26, 27 and 28 reveals VAR results for Saudi Arabia, Kuwait, UAE, Qatar, Bahrain and Oman respectively. Evidently, the variables "Oil prices and $\triangle E P U "$ negatively respond to the GCC stock markets. Granger Casuality test is performed to test whether respective variables have predictive power to the GCC stock markets (Bai, Cui, \& Zhang, 2018). Granger Casuality analysis technique is applied to test the following hypothesis:

\section{$H 0:$ The variables Oil prices and $\triangle E P U$ do not granger cause on the stock market}

Against;

\section{$H a:$ The variables Oil prices and $\triangle E P U$ do granger cause on the stock market}

The stated null hypothesis is rejected when p-value is statistically significant (set pvalue $=0.05$ ). The illustration on table 5 reveals granger Casuality analysis for Saudi Arabia stock market. The p-values for the variables "oil prices and $\triangle E P U$ " are statistically insignificant at 0.05 alpha level. In this case, the stated null hypothesis is not rejected thus oil prices and $\triangle E P U$ granger causes on Saudi Arabia stock market. Secondly, the illustration on table 6 reveals granger Casuality test for Kuwait stock market. Evidently, oil prices granger causes on the stock market and also USEPU granger cause on Saudi Arabia stock market returns. The illustration on table 7 reveals granger Casuality test on UAE stock market. Oil prices granger do not granger cause on the stock market ( $p$ value $>0.05$ ) and likewise to $\triangle U S E P U(p$-value=0.183) do not cause on UAE stock market. The illustration on table 8 reveals Granger Casuality analysis on Qatar stock market. The variables oil prices and $\triangle$ USEPU do not granger cause on the UAE stock market returns. Furthermore, the illustration on table 9 reveals granger Casuality test for Bahrain stock market returns. The variables oil prices and USEPU do not granger cause on the Bahrain stock market returns. Lastly, illustration on table 10 reveals granger Casuality test analysis on Oman stock market returns. The variables oil prices and $\Delta$ USEPU do not granger cause on the Oman stock market returns. Since the p-values are statistically insignificant at 0.05 alpha level.

Table 1: Descriptive Analysis

\begin{tabular}{llll}
\hline Variables & Mean & Std. Dev. & Obs \\
\hline Bahrain & 1285.845 & 132.7379 & 104 \\
Kuwait & 6522.611 & 733.6524 & 104 \\
Oman & 5958.577 & 682.3126 & 104 \\
Qatar & 9633.359 & 1684.787 & 104 \\
Saudi Arabia & 7403.959 & 1194.965 & 104 \\
UAE & 3779.906 & 946.3606 & 104 \\
& & & 104 \\
Brent & 82.5693 & 26.70555 & 104 \\
$\Delta$ EPU & 128.2324 & 35.24104 & 104 \\
\hline
\end{tabular}




\section{Studies in Business and Economics no. 15(1)/2020}

Table 2: Correlation Analysis

\begin{tabular}{lllllllll}
\hline & $\Delta$ EPU & Bahrain & Kuwait & Oman & Qatar & $\begin{array}{l}\text { Saudi } \\
\text { Arabia }\end{array}$ & UAE & Brent \\
\hline$\Delta$ EPU & 1.0000 & & & & & & & \\
Bahrain & -0.2176 & 1.0000 & & & & & & \\
Kuwait & -0.2559 & 0.4928 & 1.0000 & & & & \\
Oman & -0.1401 & 0.4332 & 0.5308 & 1.0000 & & & & \\
Qatar & -0.6095 & 0.1921 & 0.1173 & 0.3688 & 1.0000 & & & \\
Saudi & -0.5518 & 0.3680 & 0.4852 & 0.7704 & 0.7704 & 1.0000 & & \\
Arabia & -0.7770 & 0.2125 & 0.1359 & -0.0333 & 0.7678 & 0.6249 & 1.0000 & \\
UAE & -0.770 & & & & & & & 1.0000 \\
\hline Brent & 0.3957 & -0.1076 & 0.3680 & 0.3532 & -0.2163 & 0.1185 & -0.5578 & \\
\hline
\end{tabular}

Each of the GCC stock market monthly return is regressed on US EPU using a time series regression model shown below as:

Rit $=\alpha+\beta \mathbf{\Delta E P U i t}+$ eit

For $n=104: 1 / 31 / 2010-8 / 31 / 2018$

Table 3: OLS Regression Analysis

\begin{tabular}{llllll}
\hline Country & Coefficient & Std. Err. & $\mathbf{t}$ & Sig. & $\mathbf{R}^{\mathbf{2}}$ \\
\hline Panel: 2010:M7-2018:M8 $(\mathrm{n}=104)$ & & & & 0.000655 \\
Bahrain & -0.03468 & 0.1348 & -0.2573 & 0.7975 & 0.013831 \\
Kuwait & -1.2298 & 1.0333 & -1.1902 & 0.2368 & 0.03680 \\
Oman & -1.5460 & 0.78700 & -1.9645 & 0.0522 & 0.008772 \\
Qatar & -1.8831 & 1.9918 & -0.9454 & 0.3467 & 0.003547 \\
Saudi Arabia & -0.9708 & 1.6191 & -0.5996 & 0.5501 & 0.001546 \\
UAE & -0.2626 & 0.6641 & -0.3955 & 0.6933 & \\
\hline
\end{tabular}

In this case, the analysis applies the variables "oil prices" as a control variable primarily to have control over the US stock markets. Each GCC stock market is set as response variable and regressed on changes in EPU and oil price returns.

Rit $=\alpha+\beta \Delta E P U i t+\delta O P i t+\varepsilon i t$

Table 4: OLS with Control Variable

\begin{tabular}{lcllll}
\hline Country & Coefficient & Std. Err. & $\mathbf{t}$ & Sig. & $\mathbf{R}^{\mathbf{2}}$ \\
\hline Panel: 2010:M7-2018:M8 $(\mathrm{n}=104)$ & & & & \\
Bahrain & -0.01057 & 0.1340 & -0.0789 & 0.9372 & 0.031887 \\
Brent & 1.05497 & 0.5874 & 1.79611 & 0.0755 & 0.025083 \\
& & & & & \\
Kuwait & -1.1182 & 1.0378 & -1.077510 & & \\
& & & $-228-$ & & \\
\hline
\end{tabular}


Studies in Business and Economics no. 15(1)/2020

\begin{tabular}{|c|c|c|c|c|c|}
\hline Brent & 4.8861 & 4.5481 & 1.0743 & 0.2838 & \\
\hline Oman & -1.3336 & 0.76608 & -1.74078 & 0.0848 & \\
\hline Brent & 9.2990 & 3.357 & 2.7697 & 0.0067 & 0.1054 \\
\hline Qatar & -1.4397 & 1.9625 & 0.7336 & 0.4649 & \\
\hline Brent & 19.4040 & 8.6010 & 2.2560 & 0.0262 & 0.056778 \\
\hline Saudi Arabia & -0.3195 & 1.5003 & -0.2130 & 0.8318 & \\
\hline Brent & 28.5027 & 6.5763 & 4.3342 & 0.0000 & 0.1611 \\
\hline UAE & -0.1639 & 0.6635 & -0.24700 & 0.8054 & \\
\hline Brent & 4.3215 & 2.9077 & 1.4862 & 0.1404 & 0.023123 \\
\hline
\end{tabular}

Table 5: Granger Casuality Test Saudi Arabia

\begin{tabular}{lccc}
\hline \hline Null Hypothesis: & Obs & F-Statistic & Prob. \\
\hline \hline DIFFUSEPU does not Granger Cause DIFFSAUDI & 101 & 0.32179 & 0.7256 \\
DIFFSAUDI does not Granger Cause DIFFUSEPU & & 2.14343 & 0.1228 \\
\hline \hline DIFFBRENT does not Granger Cause DIFFSAUDI & 101 & 1.37395 & 0.2580 \\
DIFFSAUDI does not Granger Cause DIFFBRENT & & 4.80557 & 0.0103 \\
\hline \hline DIFFBRENT does not Granger Cause DIFFUSEPU & 101 & 1.19929 & 0.3059 \\
DIFFUSEPU does not Granger Cause DIFFBRENT & & 0.73998 & 0.4798 \\
\hline \hline
\end{tabular}

Table 6: Granger Casuality Test Kuwait

\begin{tabular}{lccc}
\hline \hline Null Hypothesis: & Obs & F-Statistic & Prob. \\
\hline \hline DIFFBRENT does not Granger Cause DIFFKUWAIT & 101 & 0.48738 & 0.6157 \\
DIFFKUWAIT does not Granger Cause DIFFBRENT & & 0.68763 & 0.5052 \\
\hline \hline DIFFUSEPU does not Granger Cause DIFFKUWAIT & 101 & 0.41678 & 0.6604 \\
DIFFKUWAIT does not Granger Cause DIFFUSEPU & & 1.75252 & 0.1788 \\
\hline \hline DIFFUSEPU does not Granger Cause DIFFBRENT & 101 & 0.73998 & 0.4798 \\
DIFFBRENT does not Granger Cause DIFFUSEPU & & 1.19929 & 0.3059 \\
\hline \hline
\end{tabular}




\section{Table 7: Granger Casuality Test UAE}

\begin{tabular}{lccc}
\hline \hline Null Hypothesis: & Obs & F-Statistic & Prob. \\
\hline \hline DIFFBRENT does not Granger Cause DIFFUAE & 101 & 0.19730 & 0.8213 \\
DIFFUAE does not Granger Cause DIFFBRENT & & 0.25080 & 0.7787 \\
\hline \hline DIFFUSEPU does not Granger Cause DIFFUAE & \multirow{2}{*}{01} & 0.40465 & 0.6683 \\
DIFFUAE does not Granger Cause DIFFUSEPU & & 2.73093 & 0.0702 \\
\hline \hline DIFFUSEPU does not Granger Cause DIFFBRENT & \multirow{2}{*}{01} & 0.73998 & 0.4798 \\
DIFFBRENT does not Granger Cause DIFFUSEPU & & 1.19929 & 0.3059 \\
\hline \hline
\end{tabular}

Table 8: Granger Casuality Test Qatar

\begin{tabular}{lccc}
\hline \hline Null Hypothesis: & Obs & F-Statistic & Prob. \\
\hline \hline DIFFBRENT does not Granger Cause DIFFQATAR & 101 & 0.95536 & 0.3883 \\
DIFFQATAR does not Granger Cause DIFFBRENT & & 0.24722 & 0.7815 \\
\hline \hline DIFFUSEPU does not Granger Cause DIFFQATAR & 101 & 1.48142 & 0.2325 \\
DIFFQATAR does not Granger Cause DIFFUSEPU & & 2.75027 & 0.0690 \\
\hline \hline DIFFUSEPU does not Granger Cause DIFFBRENT & 101 & 0.73998 & 0.4798 \\
DIFFBRENT does not Granger Cause DIFFUSEPU & & 1.19929 & 0.3059 \\
\hline \hline
\end{tabular}

Table 9: Granger Casuality Test Bahrain

\begin{tabular}{lccc}
\hline \hline Null Hypothesis: & Obs & F-Statistic & Prob. \\
\hline \hline DIFFBRENT does not Granger Cause DIFFBARAIN & 101 & 0.10881 & 0.8970 \\
DIFFBARAIN does not Granger Cause DIFFBRENT & & 0.68772 & 0.5052 \\
\hline \hline DIFFUSEPU does not Granger Cause DIFFBARAIN & 101 & 0.31465 & 0.7308 \\
DIFFBARAIN does not Granger Cause DIFFUSEPU & & 1.04238 & 0.3566 \\
\hline \hline DIFFUSEPU does not Granger Cause DIFFBRENT & \multirow{2}{*}{101} & 0.73998 & 0.4798 \\
DIFFBRENT does not Granger Cause DIFFUSEPU & & 1.19929 & 0.3059 \\
\hline \hline
\end{tabular}

Table 10: Granger Casuality Test Oman

\begin{tabular}{lccc}
\hline \hline Null Hypothesis: & Obs & F-Statistic & Prob. \\
\hline \hline DIFFBRENT does not Granger Cause DIFFOMAN & 101 & 0.86744 & 0.4233 \\
DIFFOMAN does not Granger Cause DIFFBRENT & & 0.29531 & 0.7450 \\
\hline \hline DIFFUSEPU does not Granger Cause DIFFOMAN & 101 & 1.15704 & 0.3188 \\
DIFFOMAN does not Granger Cause DIFFUSEPU & & 1.37438 & 0.2579 \\
\hline \hline DIFFUSEPU does not Granger Cause DIFFBRENT & 101 & 0.73998 & 0.4798 \\
DIFFBRENT does not Granger Cause DIFFUSEPU & & 1.19929 & 0.3059 \\
\hline \hline
\end{tabular}




\section{Conclusion}

The primary goal of the study was to analyze the effect of changes in economy policy uncertainty of the US to GCC countries' stock market returns. The study has applied time series OLS regression and vector autoregression analysis. The VAR has demonstrated that changes in economy policy uncertainty of US negatively responds to the GCC stock market returns. Additionally, Granger Casuality analysis was done to confirm whether economic policy uncertainty of US and oil prices granger cause on the GCC stock markets. The granger Casuality test has confirmed that changes in economic policy uncertainty of US cause the returns on the Bahrain stock market. However, this is not true on Saudi Arabia, Kuwait, UAE, Qatar and Oman stock markets. The control variable oil prices cause on the UAE and Kuwait stock markets while this cannot be said on Saudi Arabia, Qatar, Bahrain and Oman stock markets. The study is important for policymakers to better understaind how stock markets react to US policy uncertainty. The finding also will help investors in stock markets in the GCC countries.

\section{References}

Ahmed, S., Coulibaly, B., \& Zlate, A. (2017). International financial spillovers to emerging market economies: How important are economic fundamentals?. Journal of International Money and Finance, 76, 133-152.

Al-Maamary, H. M., Kazem, H. A., \& Chaichan, M. T. (2017). The impact of oil price fluctuations on common renewable energies in GCC countries. Renewable and Sustainable Energy Reviews, 75, 989-1007.

Aloui, C., Hammoudeh, S., \& ben Hamida, H. (2015). Global factors driving structural changes in the co-movement between sharia stocks and sukuk in the Gulf Cooperation Council countries. The North American Journal of Economics and Finance, 31, 311-329.

Bai, S., Cui, W., \& Zhang, L. (2018). The Granger causality analysis of stocks based on clustering. Cluster Computing, 1-6.

Bekaert, G., Ehrmann, M., Fratzscher, M., \& Mehl, A. (2014). The global crisis and equity market contagion. The Journal of Finance, 69(6), 2597-2649.

Bjørnland, H. C., \& Zhulanova, J. (2018). The Shale Oil Boom and the US Economy: Spillovers and Time-Varying Effects.

Boughanmi, H., Al-Shammakhi, A., \& Antimiani, A. (2016). Deeper Integration or Wider Integration?: the case of Gulf Cooperation Council. Journal of Economic Integration, 206-233.

Commins, D. (2015). Islam in Saudi Arabia. IB Tauris.

Duenwald, C., \& Tamirisa, N. T. (2018). Public Wage Bills in the Middle East and Central Asia. International Monetary Fund.

Gay, R. D. (2016). Effect of macroeconomic variables on stock market returns for four emerging economies: Brazil, Russia, India, and China. The International Business \& Economics Research Journal (Online), 15(3), 119.

Juselius, K. (2006). The cointegrated VAR model: methodology and applications. Oxford university press.

Mahedi Masuduzzaman (2012) "Impact of the Macroeconomic Variables on the Stock Market Returns: The Case of Germany and the United Kingdom", Global Journal of Management and Business Research. 


\section{Studies in Business and Economics no. 15(1)/2020}

Mohamed Arouri \& Christophe Rault \& Frédéric Teulon, 2014. "Economic policy uncertainty, oil price shocks and GCC stock markets," Economics Bulletin, AccessEcon, vol. 34(3), pages 18221834.

Samman, A. (2015). Introduction: Money's other worlds. Finance and Society, 1(2), 23-26.

Saxena, S., \& Al-Hadrami, A. S. N. (2017). Do We Need a GCC Bank to Facilitate the Economic Turnaround of the GCC Region?. Digest of Middle East Studies, 26(2), 226-247.

\section{Appendix}

\section{Table 11: Saudi Arabia GCC}

Dependent Variable: DIFFSAUDIARABIA

Method: Least Squares

Date: 11/26/18 Time: 10:55

Sample (adjusted): 1103

Included observations: 103 after adjustments

\begin{tabular}{lrllr}
\hline \hline \multicolumn{1}{c}{ Variable } & Coefficient & Std. Error & t-Statistic & Prob. \\
\hline \hline \multicolumn{1}{c}{ C } & -15.96543 & 41.33486 & -0.386246 & 0.7001 \\
\multicolumn{1}{c}{ DIFFUSEPU } & -0.970768 & 1.619119 & -0.599566 & 0.5501 \\
\hline \hline R-squared & 0.003547 & Mean dependent var & -16.46311 \\
Adjusted R-squared & -0.006319 & S.D. dependent var & 418.0994 \\
S.E. of regression & 419.4184 & Akaike info criterion & 14.93484 \\
Sum squared resid & 17767089 & Schwarz criterion & 14.98600 \\
Log likelihood & -767.1443 & Hannan-Quinn criter. & 14.95556 \\
F-statistic & 0.359479 & Durbin-Watson stat & 1.738374 \\
Prob(F-statistic) & 0.550138 & & & \\
\hline \hline
\end{tabular}

Table 12: Kuwait GCC Stock Market

Dependent Variable: DIFFKUWAIT

Method: Least Squares

Date: 11/26/18 Time: 10:56

Sample (adjusted): 1103

Included observations: 103 after adjustments

\begin{tabular}{lrlll}
\hline \hline \multicolumn{1}{c}{ Variable } & Coefficient & Std. Error & t-Statistic & Prob. \\
\hline \hline \multicolumn{1}{c}{ C } & 1.062596 & 26.38028 & 0.040280 & 0.9679 \\
\multicolumn{1}{c}{ DIFFUSEPU } & -1.229839 & 1.033336 & -1.190163 & 0.2368 \\
\hline \hline R-squared & 0.013831 & Mean dependent var & 0.432102 \\
Adjusted R-squared & 0.004067 & S.D. dependent var & 268.2225 \\
S.E. of regression & 267.6766 & Akaike info criterion & 14.03666 \\
Sum squared resid & 7236727. & Schwarz criterion & 14.08782 \\
Log likelihood & -720.8881 & Hannan-Quinn criter. & 14.05738 \\
F-statistic & 1.416488 & Durbin-Watson stat & 1.685124 \\
Prob(F-statistic) & 0.236772 & & \\
\hline \hline
\end{tabular}




\section{Table 13: UAE GCC Stock Market}

Dependent Variable: DIFFUAE

Method: Least Squares

Date: $11 / 26 / 18$ Time: $10: 57$

Sample (adjusted): 1103

Included observations: 103 after adjustments

\begin{tabular}{lrrrr}
\hline \hline \multicolumn{1}{c}{ Variable } & Coefficient & Std. Error & t-Statistic & Prob. \\
\hline \hline \multicolumn{1}{c}{ C } & -22.71498 & 16.95341 & -1.339847 & 0.1833 \\
\multicolumn{1}{c}{ DIFFUSEPU } & -0.262617 & 0.664078 & -0.395460 & 0.6933 \\
\hline \hline R-squared & 0.001546 & Mean dependent var & -22.84961 \\
Adjusted R-squared & -0.008340 & S.D. dependent var & 171.3107 \\
S.E. of regression & 172.0236 & Akaike info criterion & 13.15237 \\
Sum squared resid & 2988804 & Schwarz criterion & 13.20353 \\
Log likelihood & -675.3469 & Hannan-Quinn criter. & 13.17309 \\
F-statistic & 0.156389 & Durbin-Watson stat & 2.324530 \\
Prob(F-statistic) & 0.693337 & & & \\
\hline \hline
\end{tabular}

\section{Table 14: Qatar GCC Stock Market}

Dependent Variable: DIFFQATAR

Method: Least Squares

Date: $11 / 26 / 18$ Time: 10:58

Sample (adjusted): 1103

Included observations: 103 after adjustments

\begin{tabular}{lrllr}
\hline \hline \multicolumn{1}{c}{ Variable } & Coefficient & Std. Error & t-Statistic & Prob. \\
\hline \multicolumn{1}{c}{ C } & -31.34527 & 50.84968 & -0.616430 & 0.5390 \\
\multicolumn{1}{c}{ DIFFUSEPU } & -1.883135 & 1.991822 & -0.945433 & 0.3467 \\
\hline \hline R-squared & 0.008772 & Mean dependent var & -32.31068 \\
Adjusted R-squared & -0.001042 & S.D. dependent var & 515.6953 \\
S.E. of regression & 515.9639 & Akaike info criterion & 15.34918 \\
Sum squared resid & 26888090 & Schwarz criterion & 15.40034 \\
Log likelihood & -788.4826 & Hannan-Quinn criter. & 15.36990 \\
F-statistic & 0.893844 & Durbin-Watson stat & 2.310149 \\
Prob(F-statistic) & 0.346694 & & & \\
\hline \hline
\end{tabular}

\section{Table 15: Bahrain GCC Stock Market}

\section{Dependent Variable: DIFFBAHRAIN}

Method: Least Squares

Date: $11 / 26 / 18$ Time: $10: 53$

Sample (adjusted): 1103

Included observations: 103 after adjustments

\begin{tabular}{lrlll}
\hline \hline \multicolumn{1}{c}{ Variable } & Coefficient & Std. Error & t-Statistic & Prob. \\
\hline \hline \multicolumn{1}{c}{ C } & 1.378266 & 3.441593 & 0.400473 & 0.6897 \\
\multicolumn{1}{c}{ DIFFUSEPU } & -0.034683 & 0.134810 & -0.257274 & 0.7975 \\
\hline \hline R-squared & 0.000655 & Mean dependent var & 1.360485 \\
Adjusted R-squared & -0.009240 & S.D. dependent var & 34.76109 \\
S.E. of regression & 34.92131 & Akaike info criterion & 9.963298 \\
Sum squared resid & 123169.3 & Schwarz criterion & 10.01446 \\
Log likelihood & -511.1098 & Hannan-Quinn criter. & 9.984020 \\
F-statistic & 0.066190 & Durbin-Watson stat & 1.355015 \\
Prob(F-statistic) & 0.797491 & & & \\
\hline \hline
\end{tabular}




\section{Table 16: Oman GCC Stock Market}

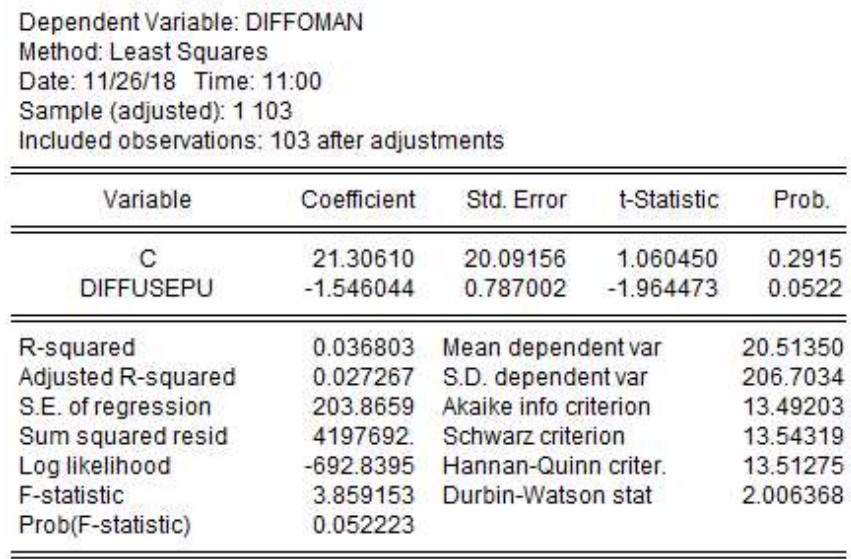

\section{Table 17: Saudi Arabia}

Dependent Variable: DIFFSAUDIARABIA

Method: Least Squares

Date: 11/26/18 Time: 11:01

Sample (adjusted): 1103

Included observations: 103 after adjustments

\begin{tabular}{lrllr}
\hline \hline \multicolumn{1}{c}{ Variable } & Coefficient & Std. Error & t-Statistic & Prob. \\
\hline \hline \multicolumn{1}{c}{ C } & -14.65001 & 38.11622 & -0.384351 & 0.7015 \\
DIFFUSEPU & -0.319544 & 1.500537 & -0.212953 & 0.8318 \\
$\quad$ DIFFBRENT & 28.50267 & 6.576250 & 4.334182 & 0.0000 \\
\hline \hline R-squared & 0.161130 & Mean dependent var & -16.46311 \\
Adjusted R-squared & 0.144352 & S.D. dependent var & 418.0994 \\
S.E. of regression & 386.7471 & Akaike info criterion & 14.78211 \\
Sum squared resid & 14957333 & Schwarz criterion & 14.85885 \\
Log likelihood & -758.2788 & Hannan-Quinn criter. & 14.81319 \\
F-statistic & 9.603957 & Durbin-Watson stat & 2.011597 \\
Prob(F-statistic) & 0.000153 & & & \\
\hline \hline
\end{tabular}

\section{Table 18: Kuwait}

\begin{tabular}{|c|c|c|c|c|}
\hline Variable & Coefficient & Std. Error & t-Statistic & Prob. \\
\hline c & 1.288095 & 26.36100 & 0.048864 & 0.9611 \\
\hline DIFFUSEPU & -1.118201 & 1.037764 & -1.077510 & 0.2838 \\
\hline DIFFBRENT & 4.886137 & 4.548104 & 1.074324 & 0.2853 \\
\hline R-squared & 0.025083 & \multicolumn{2}{|c|}{ Mean dependent var } & 0.432102 \\
\hline Adjusted R-squared & 0.005585 & \multicolumn{2}{|c|}{ S.D. dependentvar } & 268.2225 \\
\hline S.E. of regression & 267.4725 & \multicolumn{2}{|c|}{ Akaike info criterion } & 14.04460 \\
\hline Sum squared resid & 7154155 . & \multicolumn{2}{|c|}{ Schwarz criterion } & 14.12134 \\
\hline Log likelihood & -720.2971 & \multirow{2}{*}{\multicolumn{2}{|c|}{$\begin{array}{l}\text { Hannan-Quinn criter. } \\
\text { Durbin-Watson stat }\end{array}$}} & 14.07569 \\
\hline F-statistic & 1.286411 & & & 1.694348 \\
\hline Prob(F-statistic) & 0.280792 & & & \\
\hline
\end{tabular}




\section{Table 19: UAE}

Dependent Variable: DIFFUAE

Method: Least Squares

Date: $11 / 26 / 18$ Time: 11:02

Sample (adjusted): 1103

Included observations: 103 after adjustments

\begin{tabular}{lrrrr}
\hline \hline \multicolumn{1}{c}{ Variable } & Coefficient & Std. Error & t-Statistic & Prob. \\
\hline \hline \multicolumn{1}{c}{ C } & -22.51554 & 16.85339 & -1.335965 & 0.1846 \\
\multicolumn{1}{c}{ DIFFUSEPU } & -0.163880 & 0.663474 & -0.247002 & 0.8054 \\
$\quad$ DIFFBRENT & 4.321501 & 2.907742 & 1.486205 & 0.1404 \\
\hline \hline R-squared & 0.023123 & Mean dependent var & -22.84961 \\
Adjusted R-squared & 0.003586 & S.D. dependent var & 171.3107 \\
S.E. of regression & 171.0033 & Akaike info criterion & 13.14994 \\
Sum squared resid & 2924214 & Schwarz criterion & 13.22668 \\
Log likelihood & -674.2217 & Hannan-Quinn criter. & 13.18102 \\
F-statistic & 1.183533 & Durbin-Watson stat & 2.351624 \\
Prob(F-statistic) & 0.310447 & & & \\
\hline \hline
\end{tabular}

Table 20: Qatar

Dependent Variable: DIFFQATAR

Method: Least Squares

Date: $11 / 26 / 18$ Time: 11:03

Sample (adjusted): 1103

Included observations: 103 after adjustments

\begin{tabular}{lrrrr}
\hline \hline \multicolumn{1}{c}{ Variable } & Coefficient & Std. Error & t-Statistic & Prob. \\
\hline \hline \multicolumn{1}{c}{ C } & -30.44975 & 49.85204 & -0.610803 & 0.5427 \\
$\quad$ DIFFUSEPU & -1.439795 & 1.962545 & -0.733636 & 0.4649 \\
$\quad$ DIFFBRENT & 19.40401 & 8.601049 & 2.256005 & 0.0262 \\
\hline \hline R-squared & 0.056778 & Mean dependent var & -32.31068 \\
Adjusted R-squared & 0.037914 & S.D. dependent var & 515.6953 \\
S.E. of regression & 505.8249 & Akaike info criterion & 15.31895 \\
Sum squared resid & 25585881 & Schwarz criterion & 15.39569 \\
Log likelihood & -785.9260 & Hannan-Quinn criter. & 15.35003 \\
F-statistic & 3.009798 & Durbin-Watson stat & 2.334140 \\
Prob(F-statistic) & 0.053789 & & & \\
\hline \hline
\end{tabular}

Table 21: Bahrain

Dependent Variable: DIFFBAHRAIN

Method: Least Squares

Date: $11 / 26 / 18$ Time: $11: 03$

Sample (adjusted): 1103

Included observations: 103 after adjustments

\begin{tabular}{lrlrr}
\hline \hline \multicolumn{1}{c}{ Variable } & Coefficient & Std. Error & t-Statistic & Prob. \\
\hline \hline \multicolumn{1}{c}{ C } & 1.426954 & 3.404390 & 0.419151 & 0.6760 \\
DIFFUSEPU & -0.010579 & 0.134022 & -0.078936 & 0.9372 \\
DIFFBRENT & 1.054975 & 0.587365 & 1.796116 & 0.0755 \\
\hline \hline R-squared & 0.031887 & Mean dependent var & 1.360485 \\
Adjusted R-squared & 0.012524 & S.D. dependent var & 34.76109 \\
S.E. of regression & 34.54273 & Akaike info criterion & 9.950965 \\
Sum squared resid & 119320.0 & Schwarz criterion & 10.02770 \\
Log likelihood & -509.4747 & Hannan-Quinn criter. & 9.982047 \\
F-statistic & 1.646841 & Durbin-Watson stat & 1.338318 \\
Prob(F-statistic) & 0.197839 & & & \\
\hline \hline
\end{tabular}




\section{Studies in Business and Economics no. 15(1)/2020}

\section{Table 22: Oman}

Dependent Variable: DIFFOMAN

Method: Least Squares

Date: 11/26/18 Time: 11:04

Sample (adjusted): 1103

Included observations: 103 after adjustments

\begin{tabular}{lrlrr}
\hline \hline \multicolumn{1}{c}{ Variable } & Coefficient & Std. Error & t-Statistic & Prob. \\
\hline \hline \multicolumn{1}{c}{ C } & 21.73525 & 19.45979 & 1.116932 & 0.2667 \\
DIFFUSEPU & -1.333581 & 0.766082 & -1.740782 & 0.0848 \\
$\quad$ DIFFBRENT & 9.299058 & 3.357428 & 2.769697 & 0.0067 \\
\hline \hline R-squared & 0.105428 & Mean dependent var & 20.51350 \\
Adjusted R-squared & 0.087536 & S.D. dependent var & 206.7034 \\
S.E. of regression & 197.4492 & Akaike info criterion & 13.43753 \\
Sum squared resid & 3898620. & Schwarz criterion & 13.51427 \\
Log likelihood & -689.0330 & Hannan-Quinn criter. & 13.46862 \\
F-statistic & 5.892639 & Durbin-Watson stat & 2.056431 \\
Prob(F-statistic) & 0.003809 & & \\
\hline \hline
\end{tabular}

\section{Table 23: Saudi Arabia VAR Analysis}

Vector Autoregression Estimates

Date: $11 / 26 / 18$ Time: $11: 16$

Sample (adjusted): 3103

Included observations: 101 after adjustments

Standard errors in () \& t-statistics in []

\begin{tabular}{|c|c|c|c|}
\hline & DIFFSAUDIA... & DIFFUSEPU & DIFFBRENT \\
\hline DIFFSAUDIARABIA(-1) & $\begin{array}{c}0.085301 \\
(0.11016) \\
{[0.77432]}\end{array}$ & $\begin{array}{r}-0.010241 \\
(0.00641) \\
{[-1.59742]}\end{array}$ & $\begin{array}{c}0.003227 \\
(0.00147) \\
{[2.18980]}\end{array}$ \\
\hline DIFFSAUDIARABIA(-2) & $\begin{array}{r}-0.174673 \\
(0.11369) \\
{[-1.53642]}\end{array}$ & $\begin{array}{c}0.002506 \\
(0.00662) \\
{[0.37874]}\end{array}$ & $\begin{array}{r}-0.003159 \\
(0.00152) \\
{[-2.07662]}\end{array}$ \\
\hline DIFFUSEPU(-1) & $\begin{array}{c}0.124462 \\
(1.72424) \\
{[0.07218]}\end{array}$ & $\begin{array}{r}-0.293039 \\
(0.10034) \\
{[-2.92045]}\end{array}$ & $\begin{array}{r}-0.008212 \\
(0.02307) \\
{[-0.35598]}\end{array}$ \\
\hline DIFFUSEPU(-2) & $\begin{array}{r}-1.061742 \\
(1.70990) \\
{[-0.62094]}\end{array}$ & $\begin{array}{r}-0.247509 \\
(0.09951) \\
{[-2.48737]}\end{array}$ & $\begin{array}{c}0.021356 \\
(0.02288) \\
{[0.93353]}\end{array}$ \\
\hline DIFFBRENT(-1) & $\begin{array}{c}10.50060 \\
(8.12154) \\
{[1.29293]}\end{array}$ & $\begin{array}{r}-0.405538 \\
(0.47263) \\
{[-0.85805]}\end{array}$ & $\begin{array}{r}0.129905 \\
(0.10866) \\
{[1.19556]}\end{array}$ \\
\hline DIFFBRENT(-2) & $\begin{array}{c}6.728202 \\
(7.92276) \\
{[0.84922]}\end{array}$ & $\begin{array}{c}0.193877 \\
(0.46106) \\
{[0.42051]}\end{array}$ & $\begin{array}{c}0.101315 \\
(0.10600) \\
{[0.95583]}\end{array}$ \\
\hline c & $\begin{array}{r}-12.74189 \\
(41.9729) \\
{[-0.30357]}\end{array}$ & $\begin{array}{c}0.396947 \\
(2.44257) \\
{[0.16251]}\end{array}$ & $\begin{array}{c}0.042229 \\
(0.56155) \\
{[0.07520]}\end{array}$ \\
\hline quared & 0.059192 & 0.157002 & 0.132043 \\
\hline
\end{tabular}




\section{Table 24: Kuwait VAR Analysis}

\begin{tabular}{|c|c|c|c|}
\hline & DIFFKUWAIT & DIFFUSEPU & DIFFBRENT \\
\hline DIFFKUWAIT(-1) & $\begin{array}{l}0.160441 \\
(0.10337) \\
{[1.55212]}\end{array}$ & $\begin{array}{r}-0.015941 \\
(0.00935) \\
{[-1.70421]}\end{array}$ & $\begin{array}{r}-0.000136 \\
(0.00225) \\
{[-0.06071]}\end{array}$ \\
\hline DIFFKUWAIT(-2) & $\begin{array}{r}-0.016534 \\
(0.10561) \\
{[-0.15656]}\end{array}$ & $\begin{array}{r}-0.002814 \\
(0.00956) \\
{[-0.29443]}\end{array}$ & $\begin{array}{r}-0.002315 \\
(0.00230) \\
{[-1.00867]}\end{array}$ \\
\hline DIFFUSEPU(-1) & $\begin{array}{c}0.018012 \\
(1.10695) \\
{[0.01627]}\end{array}$ & $\begin{array}{r}-0.319209 \\
(0.10017) \\
{[-3.18669]}\end{array}$ & $\begin{array}{r}-0.005081 \\
(0.02406) \\
{[-0.21121]}\end{array}$ \\
\hline DIFFUSEPU(-2) & $\begin{array}{c}1.060900 \\
(1.10774) \\
{[0.95772]}\end{array}$ & $\begin{array}{r}-0.275617 \\
(0.10024) \\
{[-2.74954]}\end{array}$ & $\begin{array}{c}0.023396 \\
(0.02407) \\
{[0.97190]}\end{array}$ \\
\hline DIFFBRENT(-1) & $\begin{array}{r}3.322553 \\
(4.72695) \\
{[0.70290]}\end{array}$ & $\begin{array}{r}-0.606970 \\
(0.42775) \\
{[-1.41899]}\end{array}$ & $\begin{array}{l}0.172030 \\
(0.10272) \\
{[1.67470]}\end{array}$ \\
\hline DIFFBRENT(-2) & $\begin{array}{c}3.095637 \\
(4.75899) \\
{[0.65048]}\end{array}$ & $\begin{array}{c}0.259865 \\
(0.43065) \\
{[0.60343]}\end{array}$ & $\begin{array}{l}0.054158 \\
(0.10342) \\
{[0.52367]}\end{array}$ \\
\hline C & $\begin{array}{c}4.705591 \\
(26.8609) \\
{[0.17518]}\end{array}$ & $\begin{array}{c}0.627270 \\
(2.43068) \\
{[0.25806]}\end{array}$ & $\begin{array}{c}0.053757 \\
(0.58372) \\
{[0.09209]}\end{array}$ \\
\hline R-squ & 0.047957 & 0.161522 & 0.058028 \\
\hline
\end{tabular}

\section{Table 25: UAE VAR Analysis}

Vector Autoregression Estimates

Date: $11 / 26 / 18$ Time: $11: 19$

Sample (adjusted): 3103

Included observations: 101 after adjustments

Standard errors in () \& t-statistics in []

\begin{tabular}{cccc}
\hline \hline & DIFFUAE & DIFFUSEPU & DIFFBRENT \\
\hline \hline DIFFUAE(-1) & -0.155653 & -0.029480 & 0.001891 \\
& $(0.10369)$ & $(0.01447)$ & $(0.00351)$ \\
& {$[-1.50111]$} & {$[-2.03780]$} & {$[0.53874]$} \\
& & & \\
DIFFUAE(-2) & 0.051128 & 0.004239 & 0.001963 \\
& $(0.10741)$ & $(0.01499)$ & $(0.00364)$ \\
& {$[0.47599]$} & {$[0.28289]$} & {$[0.53981]$} \\
& & & \\
DIFFUSEPU(-1) & 0.221352 & -0.292359 & 0.000837 \\
& $(0.71830)$ & $(0.10022)$ & $(0.02431)$ \\
& {$[0.30816]$} & {$[-2.91729]$} & {$[0.03442]$} \\
DIFFUSEPU(-2) & -0.495965 & -0.250855 & 0.028050 \\
& $(0.70576)$ & $(0.09847)$ & $(0.02389)$ \\
& {$[-0.70274]$} & {$[-2.54765]$} & {$[1.17426]$} \\
& & & \\
DIFFBRENT(-1) & 1.033615 & -0.536739 & 0.167380 \\
& $(3.06317)$ & $(0.42737)$ & $(0.10368)$ \\
& {$[0.33743]$} & {$[-1.25593]$} & {$[1.61442]$} \\
& & & \\
DIFFBRENT(-2) & 1.248891 & 0.194302 & 0.032752 \\
& $(3.06911)$ & $(0.42819)$ & $(0.10388)$ \\
& {$[0.40692]$} & {$[0.45377]$} & {$[0.31529]$} \\
& & & \\
& -22.66030 & -0.008558 & 0.115779 \\
C & $(17.5980)$ & $(2.45522)$ & $(0.59563)$ \\
& {$[-1.28767]$} & {$[-0.00349]$} & {$[0.19438]$} \\
\hline \hline R-squared & 0.040570 & 0.173370 & 0.052274 \\
& & & \\
\hline
\end{tabular}




\section{Table 26: Qatar VAR Analysis}

Vector Autoregression Estimates

Date: 11/26/18 Time: 11:19

Sample (adjusted): 3103

Included observations: 101 after adjustments

Standard errors in () \& t-statistics in []

\begin{tabular}{cccc}
\hline \hline & DIFFQATAR & DIFFUSEPU & DIFFBRENT \\
\hline \hline DIFFQATAR(- 1$)$ & -0.174292 & -0.008517 & $-7.62 E-06$ \\
& $(0.10407)$ & $(0.00496)$ & $(0.00120)$ \\
& {$[-1.67483]$} & {$[-1.71824]$} & {$[-0.00633]$} \\
& & & \\
DIFFQATAR(-2) & -0.098923 & -0.007544 & -0.000699 \\
& $(0.10537)$ & $(0.00502)$ & $(0.00122)$ \\
& {$[-0.93881]$} & {$[-1.50294]$} & {$[-0.57375]$} \\
& & & \\
DIFFUSEPU(-1) & 3.894616 & -0.328253 & -0.004276 \\
& $(2.09937)$ & $(0.10000)$ & $(0.02426)$ \\
& {$[1.85514]$} & {$[-3.28248]$} & {$[-0.17623]$} \\
DIFFUSEPU(-2) & 1.489603 & -0.246668 & 0.025651 \\
& $(2.10924)$ & $(0.10047)$ & $(0.02438)$ \\
& {$[0.70623]$} & {$[-2.45509]$} & {$[1.05222]$} \\
DIFFBRENT(-1) & 1.966686 & -0.475586 & 0.176922 \\
& $(9.15016)$ & $(0.43586)$ & $(0.10576)$ \\
& {$[0.21493]$} & {$[-1.09115]$} & {$[1.67294]$} \\
& & & \\
DIFFBRENT(-2) & 13.69991 & 0.309210 & 0.054260 \\
& $(9.11853)$ & $(0.43435)$ & $(0.10539)$ \\
& {$[1.50242]$} & {$[0.71189]$} & {$[0.51485]$} \\
& & & \\
& -31.09661 & 0.112106 & 0.023060 \\
C & $(50.8652)$ & $(2.42292)$ & $(0.58789)$ \\
& {$[-0.61135]$} & {$[0.04627]$} & {$[0.03923]$} \\
\hline \hline R-squared & 0.088102 & 0.172292 & 0.050749 \\
\hline & & &
\end{tabular}

\section{Table 27: Bahrain VAR Analysis}

Vector Autoregression Estimates

Date: $11 / 26 / 18$ Time: $11: 20$

Sample (adjusted): 3103

Included observations: 101 after adjustments

Standard errors in () \& t-statistics in []

\begin{tabular}{|c|c|c|c|}
\hline & DIFFBAHRAIN & DIFFUSEPU & DIFFBRENT \\
\hline DIFFBAHRAIN(-1) & $\begin{array}{l}0.309185 \\
(0.10543) \\
{[2.93259]}\end{array}$ & $\begin{array}{c}0.050663 \\
(0.07604) \\
{[0.66627]}\end{array}$ & $\begin{array}{r}-0.019649 \\
(0.01823) \\
{[-1.07759]}\end{array}$ \\
\hline DIFFBAHRAIN(-2) & $\begin{array}{r}-0.016430 \\
(0.10654) \\
{[-0.15421]}\end{array}$ & $\begin{array}{r}-0.136396 \\
(0.07684) \\
{[-1.77505]}\end{array}$ & $\begin{array}{l}0.012552 \\
(0.01843) \\
{[0.68119]}\end{array}$ \\
\hline DIFFUSEPU(-1) & $\begin{array}{r}-0.104255 \\
(0.13746) \\
{[-0.75846]}\end{array}$ & $\begin{array}{r}-0.286967 \\
(0.09914) \\
{[-2.89462]}\end{array}$ & $\begin{array}{r}-0.003112 \\
(0.02377) \\
{[-0.13090]}\end{array}$ \\
\hline DIFFUSEPU(-2) & $\begin{array}{r}-0.061898 \\
(0.13738) \\
{[-0.45055]}\end{array}$ & $\begin{array}{r}-0.259863 \\
(0.09909) \\
{[-2.62263]}\end{array}$ & $\begin{array}{l}0.026174 \\
(0.02376) \\
{[1.10157]}\end{array}$ \\
\hline DIFFBRENT(-1) & $\begin{array}{c}0.193869 \\
(0.60503) \\
{[0.32043]}\end{array}$ & $\begin{array}{r}-0.785412 \\
(0.43637) \\
{[-1.79990]}\end{array}$ & $\begin{array}{c}0.202054 \\
(0.10464) \\
{[1.93094]}\end{array}$ \\
\hline DIFFBRENT(-2) & $\begin{array}{c}-0.265876 \\
(0.60645) \\
{[-0.43841]}\end{array}$ & $\begin{array}{c}0.355182 \\
(0.43739) \\
{[0.81204]}\end{array}$ & $\begin{array}{c}0.031415 \\
(0.10489) \\
{[0.29952]}\end{array}$ \\
\hline c & $\begin{array}{l}1.605581 \\
(3.37658) \\
{[0.47550]}\end{array}$ & $\begin{array}{c}0.710157 \\
(2.43530) \\
{[0.29161]}\end{array}$ & $\begin{array}{c}0.047779 \\
(0.58398) \\
{[0.08182]}\end{array}$ \\
\hline & 0.106120 & 0.160916 & 0.060072 \\
\hline
\end{tabular}




\section{Table 28: Oman VAR Analysis}

Vector Autoregression Estimates

Date: 11/26/18 Time: 11:20

Sample (adjusted): 3103

Included observations: 101 after adjustments

Standard errors in () \& t-statistics in []

\begin{tabular}{|c|c|c|c|}
\hline & DIFFOMAN & DIFFUSEPU & DIFFBRENT \\
\hline DIFFOMAN $(-1)$ & $\begin{array}{r}-0.005530 \\
(0.10744) \\
{[-0.05147]}\end{array}$ & $\begin{array}{r}-0.015711 \\
(0.01259) \\
{[-1.24814]}\end{array}$ & $\begin{array}{r}-0.000971 \\
(0.00301) \\
{[-0.32279]}\end{array}$ \\
\hline DIFFOMAN(-2) & $\begin{array}{r}-0.056321 \\
(0.10803) \\
{[-0.52134]}\end{array}$ & $\begin{array}{c}0.005043 \\
(0.01266) \\
{[0.39842]}\end{array}$ & $\begin{array}{r}-0.001796 \\
(0.00303) \\
{[-0.59360]}\end{array}$ \\
\hline DIFFUSEPU(-1) & $\begin{array}{c}0.950029 \\
(0.86973) \\
{[1.09232]}\end{array}$ & $\begin{array}{r}-0.306164 \\
(0.10190) \\
{[-3.00469]}\end{array}$ & $\begin{array}{r}-0.005131 \\
(0.02436) \\
{[-0.21062]}\end{array}$ \\
\hline DIFFUSEPU(-2) & $\begin{array}{c}-0.632824 \\
(0.87248) \\
{[-0.72531]}\end{array}$ & $\begin{array}{r}-0.236874 \\
(0.10222) \\
{[-2.31736]}\end{array}$ & $\begin{array}{c}0.025520 \\
(0.02444) \\
{[1.04425]}\end{array}$ \\
\hline DIFFBRENT(-1) & $\begin{array}{c}3.890391 \\
(3.78783) \\
{[1.02708]}\end{array}$ & $\begin{array}{r}-0.513075 \\
(0.44377) \\
{[-1.15617]}\end{array}$ & $\begin{array}{c}0.184094 \\
(0.10610) \\
{[1.73511]}\end{array}$ \\
\hline DIFFBRENT(-2) & $\begin{array}{c}2.342338 \\
(3.79664) \\
{[0.61695]}\end{array}$ & $\begin{array}{c}0.189498 \\
(0.44480) \\
{[0.42603]}\end{array}$ & $\begin{array}{c}0.058755 \\
(0.10635) \\
{[0.55249]}\end{array}$ \\
\hline c & $\begin{array}{c}24.09067 \\
(21.1681) \\
{[1.13806]}\end{array}$ & $\begin{array}{c}0.801142 \\
(2.47999) \\
{[0.32304]}\end{array}$ & $\begin{array}{c}0.098006 \\
(0.59293) \\
{[0.16529]}\end{array}$ \\
\hline lared & 0.041686 & 0.148526 & 0.051862 \\
\hline
\end{tabular}

ADF Tests

\begin{tabular}{|c|c|c|c|c|}
\hline \multicolumn{5}{|c|}{ Augmented Dickey-Fuller Unit Root Test on BAHRAIN } \\
\hline \multicolumn{5}{|c|}{$\begin{array}{l}\text { Null Hypothesis: BAHRAIN has a unit root } \\
\text { Exogenous: None } \\
\text { Lag Length: } 1 \text { (Automatic - based on SIC, maxlag=12) }\end{array}$} \\
\hline & & & t-Statistic & Prob.* \\
\hline Augmented Dickey-Ful & test statistic & & -0.659171 & 0.4293 \\
\hline Test critical values: & $\begin{array}{c}1 \% \text { level } \\
5 \% \text { level } \\
10 \% \text { level }\end{array}$ & & $\begin{array}{l}-2.587831 \\
-1.944006 \\
-1.614656\end{array}$ & \\
\hline \multicolumn{5}{|c|}{ *MacKinnon (1996) one-sided p-values. } \\
\hline \multicolumn{5}{|c|}{$\begin{array}{l}\text { Augmented Dickey-Fuller Test Equation } \\
\text { Dependent Variable: D(BAHRAIN) } \\
\text { Method: Least Squares } \\
\text { Date: } 11 / 26 / 18 \text { Time: } 10: 24 \\
\text { Sample (adjusted): } 3104 \\
\text { Included observations: } 102 \text { after adjustments }\end{array}$} \\
\hline Variable & Coefficient & Std. Error & t-Statistic & Prob. \\
\hline $\begin{array}{c}\text { BAHRAIN }(-1) \\
\text { D(BAHRAIN }(-1))\end{array}$ & $\begin{array}{r}-0.001670 \\
0.316604\end{array}$ & $\begin{array}{l}0.002534 \\
0.094123\end{array}$ & $\begin{array}{r}-0.659171 \\
3.363713\end{array}$ & $\begin{array}{l}0.5113 \\
0.0011\end{array}$ \\
\hline
\end{tabular}

Figure 1: Bahrain ADF Test 
Augmented Dickey-Fuller Unit Root Test on KUWAIT

Null Hypothesis: KUWAIT has a unit root

Exogenous: Non

Lag Length: 0 (Automatic - based on SIC, maxlag $=12$

\begin{tabular}{lccc}
\hline \hline & & & \\
\hline \hline Augmented Dickey-Fuller test statistic & -0.223004 & 0.6036 \\
\hline Test critical values: & $1 \%$ level & Prob. & \\
& $5 \%$ level & -2.587607 & \\
& $10 \%$ level & -1.943974 & \\
& -1.614676 & \\
\hline
\end{tabular}

*MacKinnon (1996) one-sided p-values.

Augmented Dickey-Fuller Test Equation

Dependent Variable: D(KUWAIT)

Method: Least Squares

Date: $11 / 26 / 18$ Time: $10 \cdot 27$

Sample (adjusted): 2104

Included observations: 103 after adjustments

\begin{tabular}{ccccc}
\hline \hline Variable & Coefficient & Std. Error & t-Statistic & Prob. \\
\hline \hline KUWAIT(-1) & -0.000898 & 0.004028 & -0.223004 & 0.8240 \\
\hline \hline D nainand & nnnn105 & Mnanninnanninntionr & n.13010n \\
\hline
\end{tabular}

\section{Figure 2: Kuwait ADF Test}

Augmented Dickey-Fuller Unit Root Test on UAE

Null Hypothesis: UAE has a unit root

Exogenous: None

Lag Length: 0 (Automatic - based on SIC, maxlag $=12$ )

\begin{tabular}{lrrr}
\hline \hline & t-Statistic & Prob. ${ }^{*}$ \\
\hline \hline Augmented Dickey-Fuller test statistic & 1.099156 & 0.9287 \\
\hline Test critical values: & $1 \%$ level & -2.587607 & \\
& $5 \%$ level & -1.943974 & \\
& $10 \%$ level & -1.614676 & \\
\hline
\end{tabular}

*MacKinnon (1996) one-sided p-values.

Augmented Dickey-Fuller Test Equation

Dependent Variable: D(UAE)

Method: Least Squares

Date: 11/26/18 Time: $10 \cdot 27$

Sample (adjusted): 2104

Included observations: 103 after adjustments

\begin{tabular}{ccccc}
\hline \hline Variable & Coefficient & Std. Error & t-Statistic & Prob. \\
\hline \hline UAE $(-1)$ & 0.004792 & 0.004360 & 1.099156 & 0.2743 \\
\hline \hline
\end{tabular}

Figure 3: UAE ADF Test

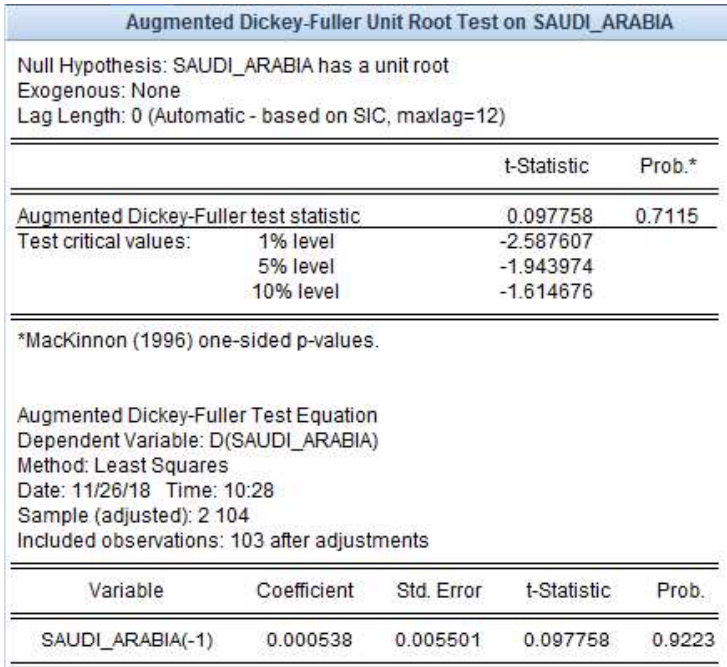

Figure 3: Saudi Arabia ADF Test 
Augmented Dickey-Fuller Unit Root Test on QATAR

Null Hypothesis: QATAR has a unit root

Exogenous: None

Lag Length: 0 (Automatic - based on SIC, maxlag=12)

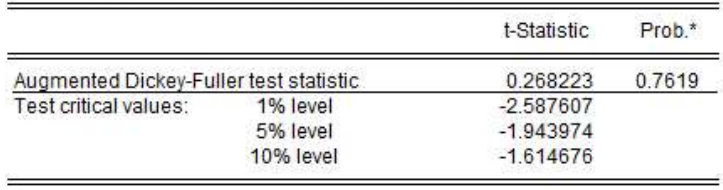

*MacKinnon (1996) one-sided p-values.

Augmented Dickey-Fuller Test Equation

Dependent Variable: D(QATAR)

Method: Least Squares

Date: $11 / 26 / 18$ Time: $10: 29$

Sample (adjusted): 2104

Included observations: 103 after adjustments

\begin{tabular}{ccccc}
\hline \hline Variable & Coefficient & Std. Error & t-Statistic & Prob. \\
\hline \hline QATAR(-1) & 0.001396 & 0.005206 & 0.268223 & 0.7891 \\
\hline
\end{tabular}

Figure 4: Qatar ADF Test

Augmented Dickey-Fuller Unit Root Test on OMAN

Null Hypothesis: OMAN has a unit roo

Exogenous: None

Lag Length: 0 (Automatic - based on SIC, maxlag=12

\begin{tabular}{lccc}
\hline \hline & & t-Statistic & Prob. $^{*}$ \\
\hline \hline \multicolumn{2}{l}{ Augmented Dickey-Fuller test statistic } & -1.093078 & 0.2472 \\
\hline Test critical values: & 1\% level & -2.587607 & \\
& $5 \%$ level & -1.943974 & \\
& 10\% level & -1.614676 & \\
\hline \hline
\end{tabular}

*Mackinnon (1996) one-sided p-values.

Augmented Dickey-Fuller Test Equation

Dependent Variable: D(OMAN)

Method: Least Squares

Date: $11 / 26 / 18$ Time: $10: 30$

Sample (adjusted). 2104

Included observations: 103 after adjustments

\begin{tabular}{ccccc}
\hline \hline Variable & Coefficient & Std. Error & t-Statistic & Prob. \\
\hline \hline OMAN(-1) & -0.003701 & 0.003386 & -1.093078 & 0.2769 \\
\hline
\end{tabular}

Figure 5: Oman ADF Test

Augmented Dickey-Fuller Unit Root Test on US_EPU

Null Hypothesis: US_EPU has a unit root

Exogenous: None

Lag Length: 3 (Automatic - based on SIC, maxlag=12)

\begin{tabular}{lccc}
\hline \hline & t-Statistic & Prob. $^{*}$ \\
\hline \hline Augmented Dickey-Fuller test statistic & -0.650984 & 0.4329 \\
\hline Test critical values: & $1 \%$ level & -2.588292 & \\
& $5 \%$ level & -1.944072 & \\
& $10 \%$ level & -1.614616 & \\
\hline \hline
\end{tabular}

*MacKinnon (1996) one-sided p-values.

Augmented Dickey-Fuller Test Equation

Dependent Variable: D(US_EPU)

Method: Least Squares

Date: 11/26/18 Time: 10:32

Sample (adjusted): 5104

Included observations: 100 after adjustments

\begin{tabular}{ccccc}
\hline \hline Variable & Coefficient & Std. Error & t-Statistic & Prob. \\
\hline \hline US_EPU(-1) & -0.011507 & 0.017677 & -0.650984 & 0.5166
\end{tabular}

Figure 6: USEPU ADF Test 
Studies in Business and Economics no. 15(1)/2020

Augmented Dickey-Fuller Unit Root Test on BRENT

Null Hypothesis: BRENT has a unit root

Exogenous: None

Lag Length: 0 (Automatic - based on SIC, maxlag=12)

\begin{tabular}{lccc}
\hline \hline & t-Statistic & Prob. $^{\star}$ \\
\hline \hline \multicolumn{2}{l}{ Augmented Dickey-Fuller test statistic } & -0.252787 & 0.5928 \\
\hline Test critical values: & $1 \%$ level & -2.587607 & \\
& $5 \%$ level & -1.943974 & \\
& $10 \%$ level & -1.614676 & \\
\hline \hline
\end{tabular}

*Mackinnon (1996) one-sided p-values.

Augmented Dickey-Fuller Test Equation

Dependent Variable: D(BRENT)

Method: Least Squares

Date: $11 / 26 / 18$ Time: $10: 32$

Sample (adjusted): 2104

Included observations: 103 after adjustments

\begin{tabular}{ccccc}
\hline \hline Variable & Coefficient & Std. Error & t-Statistic & Prob. \\
\hline \hline BRENT(-1) & -0.001678 & 0.006640 & -0.252787 & 0.8009 \\
\hline
\end{tabular}

Figure 6: Brent (Oil Prices) ADF Test

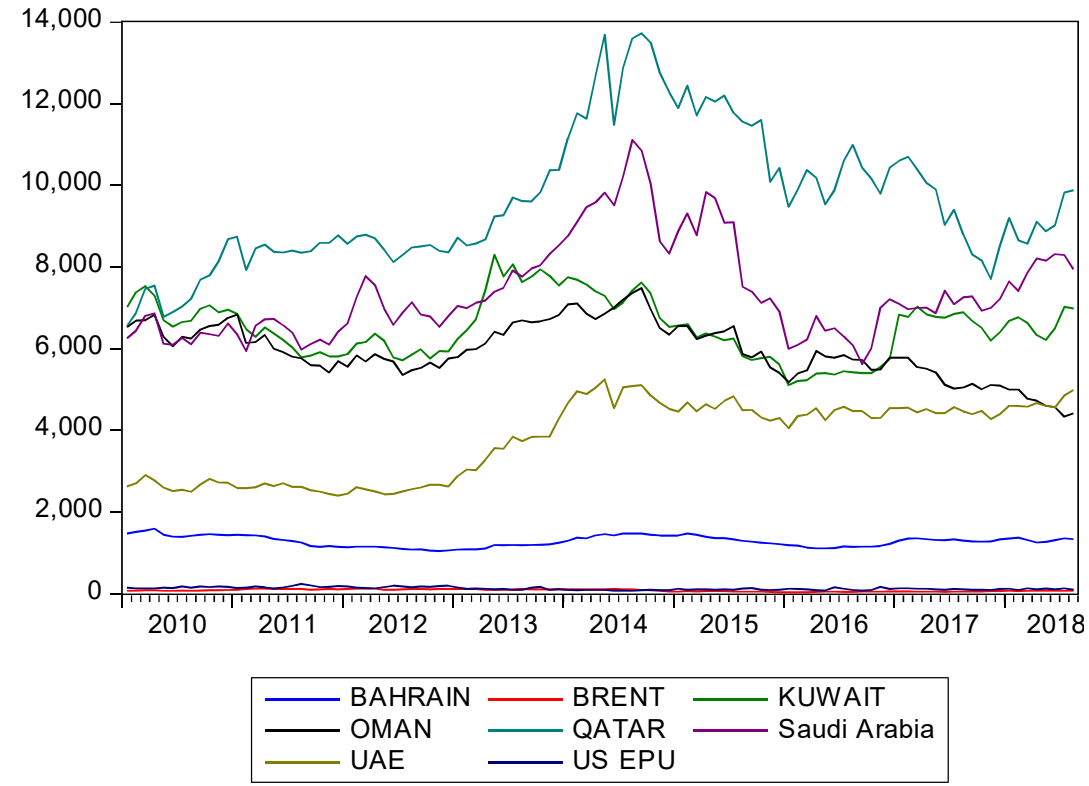

Figure 7: Multiple Line Plot 